\title{
Role of Oxidative Stress Response and Trehalose Accumulation in the Longevity of Fission Yeast
}

\author{
Bedia Palabiyik $^{1, *}$; Farinaz Jafari Ghods ${ }^{1}$ \\ ${ }^{1}$ Department of Molecular Biology and Genetics, Faculty of Science, Istanbul University, Istanbul, Turkey \\ ${ }^{*}$ Corresponding author: Bedia Palabiyik, Department of Molecular Biology and Genetics, Faculty of Science, Istanbul University, Istanbul, Turkey. Tel: +90-2124555700, \\ E-mail: bediag@istanbul.edu.tr
}

Received: December 13, 2013; Revised: May 14, 2014; Accepted: May 31, 2014

\begin{abstract}
Background: Glucose is the preferred carbon and energy source in most organisms and plays an active role in the regulation of many biological processes. However, an excess of glucose leads to such undesirable conditions as diabetes and age-related diseases. Since Schizosaccharomyces pombe homologous of many human genes, it offers several advantages for the investigation of the molecular mechanisms underlying human disease and aging studies. We have identified two glucose-repression-resistant mutants (ird5 and ird11) of S. pombe.

Objectives: We aimed to investigate the possible relationship between lifespan extension and oxidative stress response induced by exposure to hydrogen peroxide alongside the trehalose accumulation level by using the two S. pombe mutants (i.e. ird5 and ird11), which are repressed by glucose and are resistant to oxidative stress.

Materials and Methods: We employed trehalose accumulation measurement and colony-forming unit (CFU) counting using the ird mutants in exponential and stationary phases and compared them to the wild type grown in repressed, de-repressed, and stressed conditions to clarify the possible relationship between glucose signaling, oxidative stress response, and lifespan in S.pombe.

Results: The lifespan of the ird5 mutant was significantly longer that of either the ird11 mutant or the wild type cells. Under repressed condition, the trehalose content was increased remarkably on the 3rd day of the study in the ird11 mutant and the wild type. Under de-repressed condition, the level of intracellular trehalose was notably increased on the 3rd day in ird11. Under stressed condition, the trehalose level in ird11 was increased on the 3rd day as a pattern similar to that observed in the wild type.

Conclusions: Our results demonstrated no significant correlation between the ird5 lifespan and the trehalose concentration. Likewise, the correlation between lifespan extension, trehalose accumulation, and cellular resistance to hydrogen peroxide was not significant.
\end{abstract}

Keywords: Trehalose; Glucose; Repression; Fission Yeast

\section{Background}

Aging and lifespan extension have recently received considerable attention the world over. Earlier studies have reported that either caloric restriction or the inactivation of nutrient-dependent pathways is able to increase life extension in different eukaryotes. The extension of lifespan in various organisms is associated with increased resistance to oxidative stress (1). The findings of numerous studies using various models of organisms have provided indirect evidence for the hypothesis that reactive oxygen species (ROS) production and subsequent induction of ROS defense are essential contributors to longevity (2).

As in higher eukaryotes, the unicellular yeast, Saccharomyces cerevisiae, has been the most used yeast model in aging studies $(3,4)$. Yeast lifespan can be measured through two methods: replicative aging refers to the number of divisions a single mother cell undergoes before death, whereas chronological lifespan measures the viability of cultures at the stationary phase of the growth curve (5, 6 ). The fission yeast, Schizosaccharomyces pombe, has been recently used as a model for chronological aging studies inasmuch as it is more similar to the last common ancestor of humans and fungi (7). Nutrient restriction increases resistance to oxidative stress, reduces macromolecular damage, and promotes lifespan in S. pombe (8-10). Roux et al. (11) revealed that the S. pombe gpa $2^{\mathrm{R} 176 \mathrm{H}}$ mutant exhibits not only a short lifespan but also impaired mitochondrial regulation and high production of ROS. Zuin et al. (12) reported that calorie restriction favors oxidative metabolism, ROS production, and Sty1 MAP kinase activation and this stress pathway favors lifespan extension.

In S. pombe, glucose, which is a primary carbon source, is detected by $\mathrm{G}$ protein-coupled receptors and generates a signal via the cAMP-dependent protein kinase A (PKA) $(13,14)$. The glucose sensing and signaling pathways have also been found to be involved in metabolic adaptation and cellular response to diverse stress agents $(15,16)$. In $S$. pombe, the multistep phosphorelay system and the mitogen-activated protein kinase (MAPK) pathway govern the transcriptional regulation in response to oxidative stress,

Copyright (C) 2015, Ahvaz Jundishapur University of Medical Sciences. This is an open-access article distributed under the terms of the Creative Commons Attribution-NonCommercial 4.0 International License (http://creativecommons.org/licenses/by-nc/4.0/) which permits copy and redistribute the material just in noncommercial usages, provided the original work is properly cited. 
which is generated by the accumulation of ROS (17). MAPK Sty1 plays an important role in the regulation of downstream targets through triggering two transcriptional activators, Atf1 and Pap1, in oxidative stress response (18). These transcription factors activate or induce the expression of antioxidant genes such as ctt1 (cytoplasmic catalase), gpx1 (glutathione peroxidase), ttr1 (thioredoxin reductase), trx2 (thioredoxin), $n t p 1^{+}$(neutral trehalase), pgr1 (glutathione reductase), and sod1 (superoxide dismutase, SOD) (19).

Trehalose ( $\alpha, \alpha$-1, 1-diglucose) is a storage disaccharide and is present in particularly high concentrations in resting and stressed yeast cells (20). Trehalose 6-phosphate (T6P) is synthesized in S. pombe from glucose 6-phosphate and UDP-glucose by T6P synthetase, encoded by the $t p s 1^{+}$ gene (21), and converted to trehalose by T6P phosphatase, encoded by the tpp $1^{+}$gene (22). The breakdown of trehalose to glucose is catalyzed by the enzyme neutral trehalase, encoded by the $n t p 1^{+}$gene (23). Generally in yeast, the regulation of trehalose synthesis and breakdown is done by cAMP-dependent phosphorylation mechanisms (24). It has been reported that the $n t p 1^{+}$expression is regulated by the pathway of protein kinase cascade activated under osmotic or oxidative stress $(23,25)$ or by the binding of the transcription factors to elements such as cAMP-response element under thermal stress (26). In the present study, ird5 and ird11 were used to evaluate whether or not trehalose contributes to survival under moderate oxidative stress.

\section{Objectives}

In a previous study, we identified two glucose-repression-resistant mutants, namely ird5 and ird11 (27). In the ird11 mutant, the oxidative stress response is affected by glucose signaling in a manner different from that caused by glucose deprivation (28). The ird5 mutant has a lower caloric intake owing to reduced glucose consumption efficiency (29). The inefficient glucose uptake in ird5 might be a cause of increased oxidative stress response. Accordingly, in the present study, through trehalose accumulation measurement and colony-forming unit (CFU) counting, we compared the ird mutants and the wild type to clarify the possible relationship between glucose signaling, oxidative stress response, and lifespan in S. pombe.

\section{Materials and Methods}

\subsection{Yeast Strains and Media}

In this study, the wild type of S. pombe Lindner liquefaciens $\left(972 \mathrm{~h}^{-}\right)$and its relative invertase mutants (i.e. ird5 and ird11), which are resistant to glucose suppression (27), were used. The selective medium consisted of $0.5 \%$ yeast extract and 3\% sucrose and $400 \mu \mathrm{g} / \mathrm{mL}$ 2-Deoxy-Dglucose (2-DOG) was developed for the ird mutants. The strains were cultured in the YE medium containing 3\% glucose (repressed condition) and $0.5 \%$ glucose (glucose starvation condition) and the YE medium containing 0.1\% glucose plus 3\% glycerol (de-repressed condition). All the chemicals were provided by Sigma-Aldrich, Germany.

\subsection{Induction of Oxidative Stress}

The exponentially growing S. pombe cells (wild type and ird5 and ird11) under repressed conditions were split into two tubes. In the experimental group, $2 \mathrm{mM}$ hydrogen peroxide $\left(\mathrm{H}_{2} \mathrm{O}_{2}\right)$ (Sigma-Aldrich, Germany) was added to the medium, resulting in a mild level of oxidative stress in the S. pombe cells (30). The control group was not exposed to oxidative stress. After one hour, both experimental and control cells were removed by centrifugation and washed with sterile distilled water so that their trehalose content could be measured.

\subsection{Lifespan Analysis}

Chronological lifespan analyses were done on the wild type and the ird mutant cells, grown under repressed and de-repressed conditions for 21 days. Usually one day (the exponential phase) after starting the cultures, and this time point was designated as day 0 , measurements were started and continued via sampling at 2-day intervals (3rd, 5th,..., 21st days of the study).

\subsection{Extraction and Assay of Trehalose}

This study was performed under three conditions: repressed; de-repressed; and stressed conditions. From each group cells, samples were collected on the 1st (the exponential phase), 3rd, and 5th (stationary phase) days of the study, and trehalose accumulation was measured for each case. Trehalose was extracted and assayed as is described by Parrou and Francois (31). The amount of the trehalose contents of the samples was measured by treatment with trehalase (Sigma-Aldrich, Germany). The amount of the generated glucose was determined enzymatically via the glucose oxidase-peroxidase system (GOD-POD assay) using a commercial kit (Fluitest@-GLU, Biocon, Germany). The data are given as mean values \pm standard deviation (SD) with ' $n$ ' denoting the number of experiments. Statistical comparisons were made using the one-way analysis of variance (ANOVA) module of GraphPad Prism 5. The differences in the mean values were considered significant when $\mathrm{P}<0.05$.

\section{Results}

\subsection{Lifespan of S. pombe Cells under Repressed and De-Repressed Conditions}

Of the strains under study, ird5 showed the most longevity in both repressed and de-repressed conditions. The ird11 mutant had a lower lifespan extension than the wild type under repressed condition, while it had a higher lifespan extension than the wild type under de-repressed condition. On the other hand, the lifespan extension of ird11 
grown in de-repressed condition was similar to that of $i r d 5$ grown in repressed or de-repressed condition (Figure 1).

\subsection{Trehalose Accumulation of S. pombe Cells under Repressed, De-Repressed, and Stressed Conditions}

To comprehend the relationship between trehalose concentrations in the cells grown in repressed, de-repressed, and stressed conditions, the level of trehalose was estimated in the wild type and the mutant cells. The intracellular trehalose concentrations during the period of growth under repressed, de-repressed, and stressed conditions relative to the wild type and mutant cells are shown in Figure 2 A, Figure 2 B, and Figure 2 C, respectively. The results obtained from trehalose content measurement in the three conditions were evaluated in two ways. First, each group was evaluated individually and the trehalose contents of the groups on the 3rd and 5th days of the study were compared with those of the 1st day. Second, the trehalose content of each group was compared with that of the wild type.

When compared independently, as is depicted in Figure 2 A, except for ird5 under repressed condition, the trehalose content was increased remarkably on the 3rd day of the study. However, a comparison with the wild type showed a sharp decrease in the trehalose level in the ird mutant. As is evident from Figure $2 \mathrm{~B}$, under de-repressed condition, the level of intracellular trehalose was notably increased in the stationary phase (3rd day), while no significant changes were detected in either ird5 or in ird11. Under stressed condition (Figure $2 \mathrm{C}$ ), during the stationary phase (3rd day), the trehalose level in ird11 was increased as a pattern similar to that observed in the wild type; nevertheless, only trace amounts of alteration were seen in ird5. Finally, concerning the wild type during the stationary growth phase, a dramatic decrease was observed in the trehalose content in $\operatorname{ird} 5$ (3rd and 5th days) and $\operatorname{ird11}$ (3rd day).

\section{Discussion}

The PKA activated by glucose starvation and stressactivated MAPK pathways regulate the transcription of downstream genes via protein-DNA interactions at UAS1 and UAS2 of the $f b p 1$ gene (32). The existence of crosstalking between Sty1p and Pka1p regulates not only glucose repression and oxidative stress response pathways but also trehalose accumulation. Paredes et al. (25) reported that the expression of the $n t p 1^{+}$and $t p s 1^{+}$genes in $S$. pombe is partially regulated by the Stylp kinase under salt-induced osmotic stress and conditions of slight oxidative stress and is fully dependent on this kinase under severe oxidative stress. Another study showed that cell viability may depend on capacity to rapidly degrade the trehalose that is accumulated during oxidative stress. The authors indicated that glutathione reductase can be inhibited by trehalose in a dose-dependent manner. On the other hand, Sanchez-Fresneda et al. (33) reported that the stress-induced trehalose accumulation is Hog1-independent in Candida albicans.

Figure 1. Lifespan of the S. pombe Wild Type and ird Mutants
A

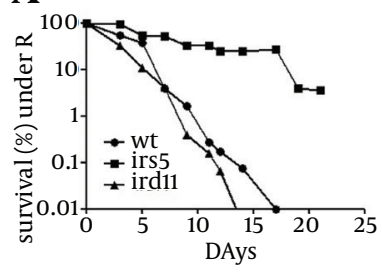

B

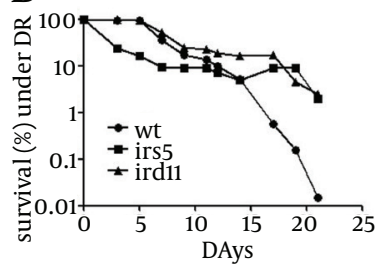

A, Grown in repressed condition (YE-3\% glucose); B, Grown in de-repressed condition (YE-0.1\% glucose plus 3\% glycerol); (wt: wild type, R: repressed, DR: de-repressed condition).
A

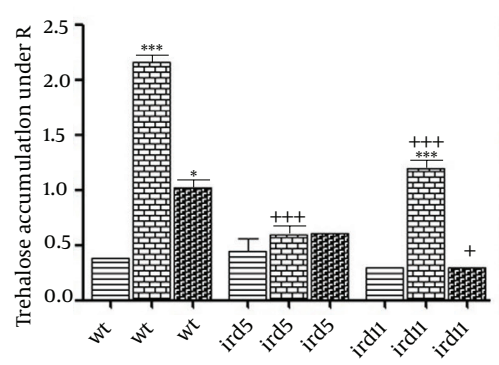

B

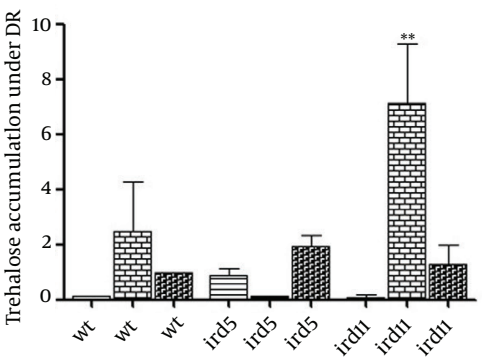

C

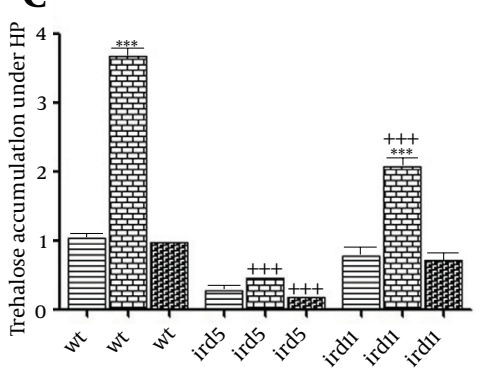

A, Grown in repressed condition (YE-3\% glucose); B, Grown in repressed condition (YE-0.1\% glucose plus 3\% glycerol); C, Grown in stressed condition (2 mM $\mathrm{H}_{2} \mathrm{O}_{2}$, one hour). The trehalose contents are expressed as mg glucose per $0.2 \mathrm{~g}$ of wet mass of the cells. Statistical significance was evaluated by one-way ANOVA (P<0.05-0,001). (wt: wild type, R: repressed, DR: de-repressed, HP: oxidative stress conditions). * , **, ***: Each group was evaluated individually and the trehalose contents of the groups on the 3rd and 5th days of the study were compared with those of the 1 st day.,,++++++ : Trehalose content of each group is compared with that of the wild type. 
The extension of lifespan in S. pombe is associated with both calorie restriction and increased resistance to oxidative stress (10-12). Chiming in with a study done on $S$. cerevisiae by Mahmud et al. (34), our results suggest that lifespan extension in $S$. pombe may not be associated with oxidative stress resistance and trehalose accumulation. The oxidative stress-resistant mutant ird11, which is affected by glucose signaling in a manner different from that caused by glucose deprivation (28), had a lower lifespan extension than the wild type under repressed condition and had a higher lifespan extension than the wild type under de-repressed condition. Indeed, the lifespan extension of ird11 grown in de-repressed condition was similar to that of ird5 grown in repressed or de-repressed condition.

We suggest that the longevity of the ird5 mutant grown in both repressed and de-repressed conditions might be, in part, in consequence of glucose depletion condition caused by a reduced glucose consumption rate in these cells compared to the ird11 mutant and the wild type. Because, contrary to ird11, there is an adaptive response to oxidative stress in ird5, caused by glucose sensing/signaling which emerges in glucose starvation (29). In light of the results of the present study, it seems that lifespan extension is mostly related to glucose sensing/signaling rather than oxidative stress response and trehalose accumulation.

\section{Acknowledgements}

We thank Prof. Guler Temizkan (Istanbul University) and Dr. Cenk Kig (Catholic University of Leuven) for their valuable suggestions.

\section{Authors' Contributions}

Bedia Palabiyik:1, Hypothesis; 2, Methodology; 3, Assessment of result and preparation of manuscript; 4 . Contribution to lab study; Farinaz Jafari Ghods: 1, Lab study; 2, preparation of manuscript.

\section{Funding/Support}

This research was supported by Istanbul University Research Fund, Project Number: 1477.

\section{References}

1. Longo VD. Mutations in signal transduction proteins increase stress resistance and longevity in yeast, nematodes, fruit flies, and mammalian neuronal cells. Neurobiol Aging. 1999; 20(5):479-86.

2. Ristow M, Schmeisser S. Extending life span by increasing oxidative stress. Free Radic Biol Med. 2011;51(2):327-36.

3. Lin SJ, Kaeberlein M, Andalis AA, Sturtz LA, Defossez PA, Culotta VC, et al. Calorie restriction extends Saccharomyces cerevisiae lifespan by increasing respiration. Nature. 2002;418(6895):344-8.

4. Fontana L, Partridge L, Longo VD. Extending healthy life spanfrom yeast to humans. Science. 2010;328(5976):321-6.

5. Fabrizio P, Longo VD. The chronological life span of Saccharomyces cerevisiae. Aging Cell.2003;2(2):73-81.

6. Steinkraus KA, Smith ED, Davis C, Carr D, Pendergrass WR, Sut- phin GL, et al. Dietary restriction suppresses proteotoxicity and enhances longevity by an hsf-1-dependent mechanism in Caenorhabditis elegans. Aging Cell. 2008;7(3):394-404.

7. Sipiczki M. Fission Yeast Phylogenesis and Evolution. In: Egel $\mathrm{R}$ editor. The Molecular Biology of Schizosaccharomyces pombe. Springer-Verlag.. Heidelberg; 2004. pp. 431-43.

8. Roux AE, Quissac A, Chartrand P, Ferbeyre G, Rokeach LA. Regulation of chronological aging in Schizosaccharomyces pombe by the protein kinases Pka1 and Sck2. Aging Cell. 2006;5(4):345-57.

9. Roux AE, Leroux A, Alaamery MA, Hoffman CS, Chartrand P, Ferbeyre $G$, et al. Pro-aging effects of glucose signaling through a $\mathrm{G}$ protein-coupled glucose receptor in fission yeast. PLoS Genet. 2009;5(3):e1000408.

10. Chen BR, Runge KW. A new Schizosaccharomyces pombe chronological lifespan assay reveals that caloric restriction promotes efficient cell cycle exit and extends longevity. Exp Gerontol. 2009;44(8):493-502.

11. Roux AE, Arseneault G, Chartrand P, Ferbeyre G, Rokeach LA. A screen for genes involved in respiration control and longevity in Schizosaccharomyces pombe. Ann NYAcad Sci. 2010;1197:19-27.

12. Zuin A, Carmona M, Morales-Ivorra I, Gabrielli N, Vivancos AP, Ayte J, et al. Lifespan extension by calorie restriction relies on the Sty1 MAP kinase stress pathway. EMBO J. 2010;29(5):981-91.

13. Welton RM, Hoffman CS. Glucose monitoring in fission yeast via the Gpa2 galpha, the git5 Gbeta and the git3 putative glucose receptor. Genetics. 2000;156(2):513-21.

14. Hoffman CS. Glucose sensing via the protein kinase A pathway in Schizosaccharomyces pombe. Biochem Soc Trans. 2005;33(Pt 1):257-60.

15. Chen D, Toone WM, Mata J, Lyne R, Burns G, Kivinen K, et al. Global transcriptional responses of fission yeast to environmental stress. Mol Biol Cell. 2003;14(1):214-29.

16. Chen D, Wilkinson CR, Watt S, Penkett CJ, Toone WM, Jones N, et al. Multiple pathways differentially regulate global oxidative stress responses in fission yeast. Mol Biol Cell. 2008;19(1):308-17.

17. Marshall CJ. MAP kinase kinase kinase, MAP kinase kinase and MAP kinase. Curr Opin Genet Dev. 1994;4(1):82-9.

18. Ikner A, Shiozaki K. Yeast signaling pathways in the oxidative stress response. Mutat Res. 2005;569(1-2):13-27.

19. Feldmann H. Yeast Molecular and Cell Biology.Germany: WileyBlackwell; 2010.

20. Walker GM. Yeast Physiology and Biotechnology.Chichester UK: John Wiley \& Sons; 1998.

21. Blazquez MA, Stucka R, Feldmann H, Gancedo C. Trehalose6-P synthase is dispensable for growth on glucose but not for spore germination in Schizosaccharomyces pombe. J Bacteriol. 1994;176(13):3895-902.

22. Franco A, Soto T, Vicente-Soler J, Guillen PV, Cansado J, Gacto M Characterization of tpp1(+) as encoding a main trehalose-6P phosphatase in the fission yeast Schizosaccharomyces pombe. $J$ Bacteriol. 2000;182(20):5880-4.

23. Soto T, Fernandez J, Dominguez A, Vicente-Soler J, Cansado J, Gacto $M$. Analysis of the ntp1+ gene, encoding neutral trehalase in the fission yeast Schizosaccharomyces pombe. Biochim Biophys Acta. 1998;1443(1-2):225-9.

24. Thevelein JM. Regulation of trehalose mobilization in fungi. Microbiol Rev. 1984;48(1):42-59.

25. Paredes V, Franco A, Soto T, Vicente-Soler J, Gacto M, Cansado J. Different roles for the stress-activated protein kinase pathway in the regulation of trehalose metabolism in Schizosaccharomyces pombe. Microbiology. 2003;149(Pt 7):1745-52.

26. Paredes V, Franco A, Madrid M, Soto T, Vicente-Soler J, Gacto M, et al. Transcriptional and post-translational regulation of neutral trehalase in Schizosaccharomyces pombe during thermal stress. Yeast. 2004;21(7):593-603.

27. Kig C, Turkel S, Temizkan G. Isolation and characterization of glucose derepressed invertase mutants from Schizosaccharomyces pombe. Biosci Biotechnol Biochem. 2005;69(12):2475-8.

28. Palabiyik B, Kig C, Pekmez M, Dalyan L, Arda N, Temizkan G. Investigation of the relationship between oxidative stress and glucose signaling in Schizosaccharomyces pombe. Biochem Genet. 2012;50(5-6):336-49. 
29. Palabiyik B, Jafari Ghods F, Onay Ucar E. Effects of glucose sensing/signaling on oxidative stress response in glucose repression mutants of Schizosaccharomyces pombe. Genet Mol Res. 2013;12(4):5046-56.

30. Quinn J, Findlay VJ, Dawson K, Millar JB, Jones N, Morgan BA, et al Distinct regulatory proteins control the graded transcriptional response to increasing $\mathrm{H}(2) \mathrm{O}(2)$ levels in fission yeast Schizosaccharomyces pombe. Mol Biol Cell. 2002;13(3):805-16.

31. Parrou JL, Francois J. A simplified procedure for a rapid and reliable assay of both glycogen and trehalose in whole yeast cells. Anal Biochem. 1997;248(1):186-8.

32. Neely LA, Hoffman CS. Protein kinase A and mitogen-activated protein kinase pathways antagonistically regulate fission yeast fbp1 transcription by employing different modes of action at two upstream activation sites. Mol Cell Biol.2000;20(17):6426-34.

33. Sanchez-Fresneda R, Guirao-Abad JP, Arguelles A, Gonzalez-Parraga P, Valentin E, Arguelles JC. Specific stress-induced storage of trehalose, glycerol and D-arabitol in response to oxidative and osmotic stress in Candida albicans. Biochem Biophys Res Commun. 2013;430(4):1334-9.

34. Mahmud SA, Hirasawa T, Shimizu H. Differential importance of trehalose accumulation in Saccharomyces cerevisiae in response to various environmental stresses. J Biosci Bioeng. 2010; 109(3):262-6. 